\title{
Opposite Dynamics of GABA and Glutamate Levels in the Occipital Cortex during Visual Processing
}

\author{
Katarzyna Kurcyus, ${ }^{1}$ Efsun Annac, ${ }^{2,3}$ ia M. Hanning, ${ }^{2,3}$ Ashley D. Harris, ${ }^{4}$ Georg Oeltzschner, ${ }^{5}$ Richard Edden, ${ }^{5}$ \\ and ${ }^{\circ}$ Valentin Riedl ${ }^{1,6}$ \\ ${ }^{1}$ Department of Neuroradiology, Klinikum rechts der Isar der Technischen Universität München, 81675 Munich, Germany, ${ }^{2}$ Department of General \\ and Experimental Psychology, Ludwig-Maximilians-Universität München, 80802 Munich, Germany, ${ }^{3}$ Graduate School of Systemic Neurosciences, \\ Ludwig-Maximilians-Universität München, 82152 Munich, Germany, ${ }^{4}$ Department of Radiology, University of Calgary, Calgary, Alberta T2N 1N4, Canada, \\ ${ }^{5}$ Department of Radiology and Radiological Science, Johns Hopkins University, Baltimore, Maryland 21287, and ${ }^{6}$ Department of Nuclear Medicine, \\ Klinikum rechts der Isar der Technischen Universität München, 81675 Munich, Germany
}

\begin{abstract}
Magnetic resonance spectroscopy (MRS) measures the two most common inhibitory and excitatory neurotransmitters, GABA and glutamate, in the human brain. However, the role of MRS-derived GABA and glutamate signals in relation to system-level neural signaling and behavior is not fully understood. In this study, we investigated levels of GABA and glutamate in the visual cortex of healthy human participants (both genders) in three functional states with increasing visual input. Compared with a baseline state of eyes closed, GABA levels decreased after opening the eyes in darkness and Glx levels remained stable during eyes open but increased with visual stimulation. In relevant states, GABA and Glx correlated with amplitude of fMRI signal fluctuations. Furthermore, visual discriminatory performance correlated with the level of GABA, but not Glx. Our study suggests that differences in brain states can be detected through the contrasting dynamics of GABA and Glx, which has implications in interpreting MRS measurements.
\end{abstract}

Key words: fMRS; GABA; glutamate; rs-fMRI; visual discriminatory performance; visual system

Significance Statement

GABA and glutamate are the two most abundant neurotransmitters in human brain. Their interaction, known as inhibitoryexcitatory balance, plays a crucial role in establishing spontaneous and stimulus-driven brain activity. Yet, the relationship between magnetic resonance spectroscopy (MRS)-derived levels of both metabolites and fMRI is still a matter of dispute. In this work, we study GABA and glutamate in three states of visual processing and in relation to fMRI and visual discriminatory performance in healthy people. We found that states of visual processing can be detected through the contrasting dynamics of GABA and glutamate and their correlation with fMRI signals. We also demonstrated that GABA, but not glutamate, in the visual system predicts visual performance. Our results provide insights into MRS-derived GABA and glutamate measurements.

\section{Introduction}

Magnetic resonance spectroscopy (MRS) is a valuable method to noninvasively study the brain's neurochemical architecture. Re-

\footnotetext{
Received May 7, 2018; revised June 22, 2018; accepted Sept. 19, 2018.

Author contributions: E.A., N.M.H., A.D.H., G.O., and R.E. edited the paper; K.K. and V.R. designed research; K.K., E.A., and N.M.H. performed research; A.D.H., G.O., and R.E. contributed unpublished reagents/analytic tools; K.K., E.A., and N.M.H. analyzed data; K.K. and V.R. wrote the paper.

V.R. was supported by Deutsche Forschungsgemeinschaft Grant ID 273427765. R.E. was supported by National Institutes of Health Grants P41EB015909, R01EB016089, R01EB023963, R01MH106564, and R21MH098228. G.0. received salary support from National Institutes of Health Grants R01EB016089, R01EB023963, R01MH106564, and R21MH098228. The behavioral experiments were conducted at the Department of General and Experimental Psychology, Ludwig-Maximilians-University of Munich. We thank Prof. Dr. Thomas Geyer and Prof. Dr. Heiner Deubel for providing equipment; and Dr. Ulrich Pilatus for providing valuable advice and constructive feedback.

The authors declare no competing financial interests.

Correspondence should be addressed to either Dr. Katarzyna Kurcyus or Dr. Valentin Riedl, Department of Neuroradiology, Klinikum rechts der Isar der Technischen Universität München, Ismaninger Strasse 22, 81675 München, Germany, E-mail: katarzyna.kurcyus@tum.de or valentin.ried@tum.de.
}

cent developments in the field have given rise to spectral editing methods (edited MRS), which have enabled simultaneous quantification of the two most important inhibitory and excitatory neurotransmitters: GABA and glutamate (Glu). While imbalances in neurotransmitter concentrations have been detected with edited MRS in patients suffering psychiatric disorders (Taylor, 2014), the significance of MRS-derived GABA and Glu levels for maintaining normal brain function is still not fully understood.

Early work suggested that the edited MRS signals from a brain region acquired in a state of rest capture baseline neurometabolite levels that relate to the amplitude of neural activation in the same area while performing a task. Most of these studies focused 
on the visual system (Mangia et al., 2007; Schaller et al., 2014; Bednarík et al., 2015). Reports consistently found that resting Glu levels in the occipital cortex positively correlated with the BOLD signal amplitude of fMRI during a checkerboard stimulation (Bednarík et al., 2015; Ip et al., 2017). For GABA, the findings have thus far been less consistent. While some studies found a negative relationship between baseline GABA in the occipital cortex and BOLD signal amplitude during checkerboard stimulation (Muthukumaraswamy et al., 2009; Donahue et al., 2010; Bednarík et al., 2015), others did not detect such an association (Apsvalka et al., 2015; Harris et al., 2015). Several studies also investigated the MRS signal in relation to task-related changes in MEG recordings. While Muthukumaraswamy et al. (2009) found a positive relationship between GABA levels and the gamma oscillation frequency in the occipital cortex, a recent study including 50 participants failed to replicate this result (Cousijn et al., 2014). These inconsistencies suggest that the relationship of GABA and Glu levels to other measures of neuronal signaling remains unclear.

Functional MRS (fMRS) tracks the dynamics of neurometabolite concentrations across brain states (Schaller et al., 2014; Apsvalka et al., 2015). In a typical fMRS framework, spectra acquired in the resting state are compared with corresponding signals collected while a task is performed. In their pioneering work, Mangia et al. (2007) identified an increase in Glu and a decrease in lactate and aspartate concentrations in the occipital cortex as a result of visual stimulation. Likewise, other groups observed an increase in the Glu concentration in the visual system when presenting a flickering checkerboard (Mangia et al., 2007; Schaller et al., 2014; Bednarík et al., 2015). On the other hand, findings regarding dynamic changes in GABA levels during a task have been less consistent. Although Mekle et al. (2017) found a GABA concentration decrease in response to visual stimulation, others have failed to detect such fluctuations (Mangia et al., 2007; Schaller et al., 2014; Bednarík et al., 2015).

The heterogeneous findings regarding MRS-derived neurotransmitter levels in relation to fMRI and MEG data might result from the fact that the two imaging modalities have thus far been obtained in noncorresponding conditions (i.e., comparing baseline MRS with task fMRI/MEG). Should MRS-derived neurotransmitter levels in the visual system exhibit complex dynamics across states, recording both signals in identical conditions would be crucial. Moreover, neurotransmitter levels in the visual system have so far only been studied in a limited range of possible functional states: while resting (defined as eyes open without receiving additional input) and during checkerboard stimulation. To the best of our knowledge, no study thus far has examined both neurotransmitters across the full functional spectrum of the visual system's states, including eyes closed, eyes open, and visual input processing.

We therefore acquired fMRS of GABA and Glu (here as Glx, a complex of Glu and glutamine [Gln]) as well as fMRI data in three relevant states of the visual system: eyes closed (CLOSED), eyes open in darkness (openDARK), and eyes open with visual checkerboard stimulation (openSTIM). Finally, we determined whether individual neurotransmitter levels in early visual regions reflect visual discrimination performance by performing two psychophysical paradigms: an orientation discrimination task and an attentional capture task as a control condition.

Table 1. Comparison between WIP \#715 and WIP \#795: group-averaged GABA $+/ \mathrm{Cr}$ concentrations and GABA+ FWHM scores in the CLOSED and openDARK conditions ${ }^{a}$

\begin{tabular}{llll}
\hline Group & WIP\#715 & WIP\#795 & t value $(\mathrm{df}=48)^{a}$
\end{tabular}

$\mathrm{GABA}+/ \mathrm{Cr}$ in CLOSED

GABA $+/ C r$ in openDARK

GABA+ FWHM in CLOSED

GABA + FWHM in openDARK

Unpaired two-sample $t$ test results.

\section{Materials and Methods}

\section{Participants and experimental design}

Fifty-five healthy volunteers $\left(\mathrm{M}_{\text {age }}=27.3, \mathrm{SD}=2.94,30\right.$ males $)$ took part in the study after providing written informed consent. The main study cohort $(N=30$, mean \pm SD age, $26.9 \pm 2.65$ years, 18 males $)$ participated in both neuroimaging and behavioral testing. For this group, neuroimaging experiment consisted of MRS and fMRI blocks of three conditions: CLOSED, openDARK, and openSTIM. MRS and fMRI data were acquired in the same session on a 3T Biograph mMR system (Siemens) using a vendor-supplied 12-channel phase-array head coil for receive and the body coil for transmit. In the CLOSED condition, participants lay down with their eyes closed without falling asleep and were asked immediately after finishing this session whether they stayed awake. In the openDARK state, they kept their eyes open in a dark scanner room; and during the openSTIM condition, they were looking at a flickering checkerboard presentation. Results of the neuroimaging experiment were replicated after merging the data of the main cohort with an independent sample collected earlier as a part of another study, referred to as replication cohort $(N=25$, mean \pm SD age, $27.4 \pm 3.14$ years, 12 males $)$. This group performed MRS and fMRI blocks in two conditions: CLOSED and openDARK, without taking part in behavioral testing.

The neuroimaging data were collected at the Faculty of Medicine of the Technical University of Munich. The behavioral data were recorded on a separate day and were collected at the Department of Experimental Psychology of the Ludwig-Maximilians-Universität München. The experimental protocols were approved by the ethical review board of the Faculty of Medicine of the Technical University of Munich and of the Faculty of Psychology and Education of the Ludwig-MaximiliansUniversität München.

\section{MR image acquisition}

\section{High resolution structural imaging}

In the beginning of MR protocol, an MP-RAGE T1-weighted anatomical image was acquired using the following parameters: TR/TE $=2300 / 2.98$ ms, flip angle $=9^{\circ}, 160$ slices covering the whole brain with gap $=0.5$ $\mathrm{mm}, \mathrm{FOV}=256 \times 256 \mathrm{~mm}^{2}$, matrix size $=256 \times 256$, slice thickness $=$ $1 \mathrm{~mm}$, resulting voxel size $=1.0 \times 1.0 \times 1.0 \mathrm{~mm}^{3}$, with a total scan time of $5 \mathrm{~min}$ and $3 \mathrm{~s}$.

\section{MRS}

MRS data in the CLOSED and openDARK conditions were collected as steady-state resting sessions with a duration equal to the length of the pulse sequence ( $8 \mathrm{~min}$ and $40 \mathrm{~s}$ for WIP \#795 and $6 \mathrm{~min}$ and $30 \mathrm{~s}$ for WIP \#715) for both main and replication study cohorts. In the openSTIM condition ( performed only by the main study cohort), interleaved blocks of task and rest (30-s-long) were presented over a length of the pulse sequence (i.e., $8 \mathrm{~min}$ and $40 \mathrm{~s}$; for further details, see Checkerboard stimulation). All blocks were acquired in counterbalanced order.

MRS was acquired using the MEGA-PRESS pulse sequence provided by Siemens. The data of main cohort were collected using the WIP \#795 package; and for the replication cohort, WIP \#715 package was used. No significant differences in MRS parameters were found between the two subgroups (Table 1). A $25 \times 25 \times 25 \mathrm{~mm}^{3}$ MRS voxel was planned in the sagittal plane of the MP-RAGE and in the axial and coronal planes of the localizer image. The voxel was centered within the visual cortex along the midline and rotated appropriately to avoid lipid signal contamination from the skull. The voxel placement is depicted in Figure $2 A$. Both WIP sequences shared the following acquisition parameters: 256 single 
A

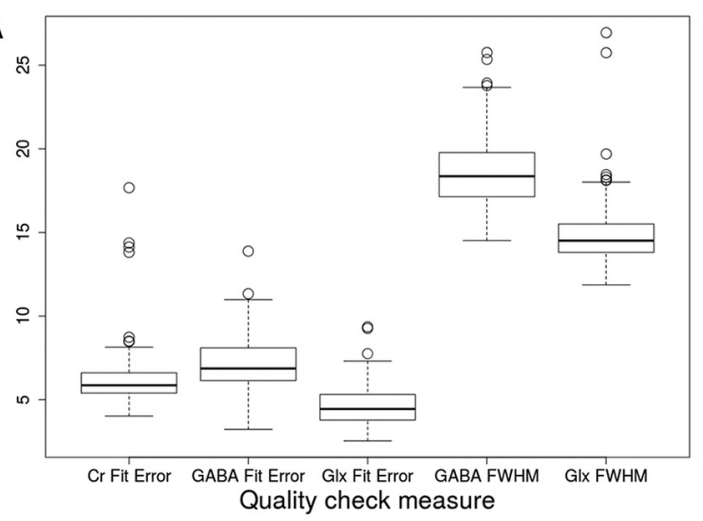

$C_{5}$
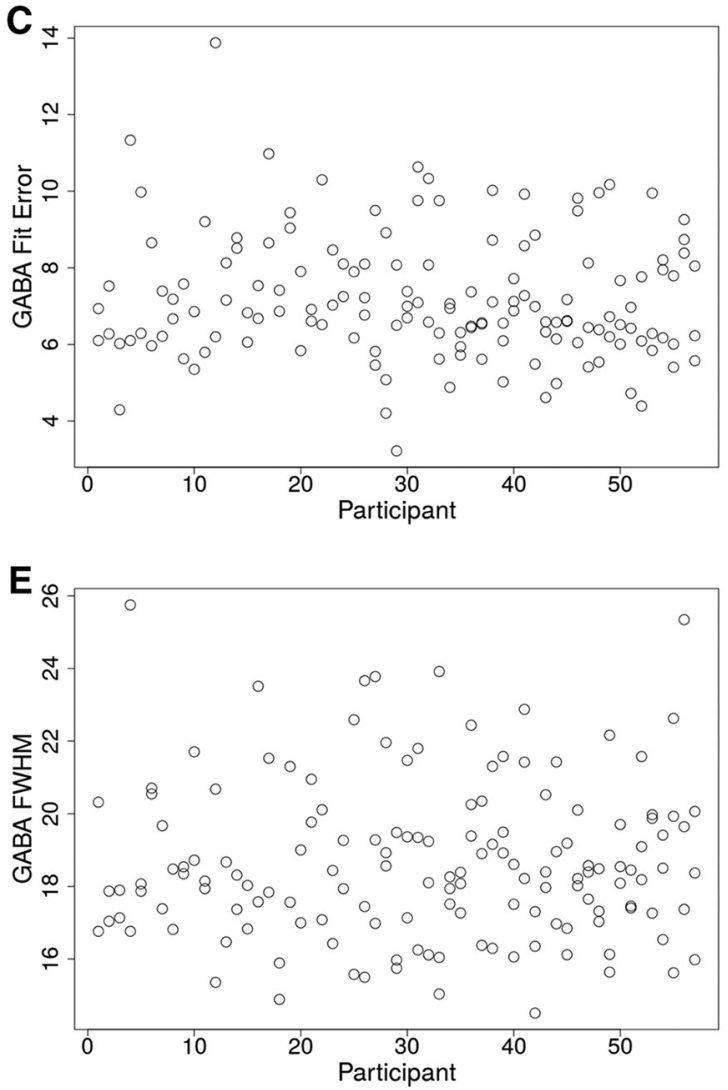
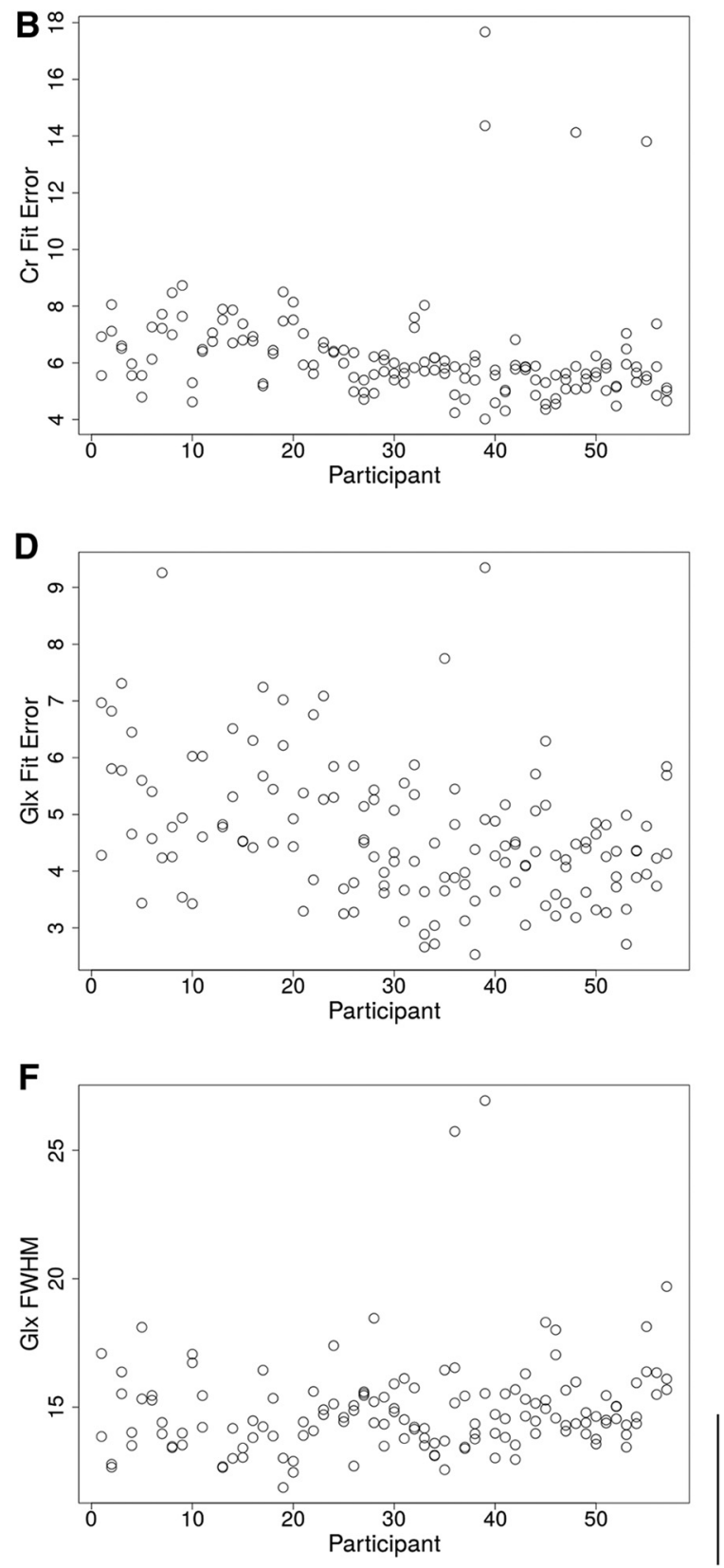

Figure 1. Single-subject parameters used to assess the quality of MRS data. $A$, Boxplots represent all parameters of interest. $B$, Scatterplot represents $C r$ fit error values. $C$, Scatterplot represents GABA + fit error values. D, Scatterplot represents Glx fit error values. E, Scatterplot represents GABA + FWHM. F, Scatterplot represents Glx FWHM.

averages (i.e., 128 edit-ON and 128 edit-OFF scans); bandwidth $=1200$ $\mathrm{Hz}$; editing J-refocusing pulses irradiated at $1.9 \mathrm{ppm}$ (edit-on) and 7.5 ppm (edit-off). The following parameters were unique for the WIP \#795 sequence: $\mathrm{TR} / \mathrm{TE}=2000 / 68 \mathrm{~ms} ; 2048$ data points; acquisition time $8 \mathrm{~min}$ and 40 s. Unique WIP \#715 parameters were as follows: TR/TE = 1500/68 ms; 512 data points; acquisition time $6 \mathrm{~min}$ and $30 \mathrm{~s}$. MRS data were acquired before EPI sequences to avoid gradient-induced frequency drifts previously described by Harris et al. (2014).

$f M R I$

fMRI resting-state data in the CLOSED and openDARK conditions were collected for both main and replication study cohorts, whereas the taskrelated openSTIM condition was performed only by the main study cohort. All blocks were acquired in counterbalanced order.

Resting-state fMRI. In the resting-state fMRI paradigm, 360 volumes of EPI images (T2*-weighted echo-planar-imaging) were acquired in interleaved fashion. Acquisition parameters were as follows: TR/TE =
$2000 / 30 \mathrm{~ms}$; flip angle $=90^{\circ} ; 35$ slices aligned along the anterior commissure/posterior commissure line and covering the whole brain; FOV $=$ $192 \times 192 \mathrm{~mm}^{2}$; matrix size $=64 \times 64$ voxels; slice thickness $=3 \mathrm{~mm}$; resulting voxel size $3.0 \times 3.0 \times 3.0 \mathrm{~mm}^{3}, 4$ dummy scans. The fMRI session lasted $12 \mathrm{~min}$ and $6 \mathrm{~s}$ and included one block of the CLOSED and one block of the openDARK condition.

Task-related fMRI. The task-related fMRI sequence, referred to as openSTIM condition, used the same fMRI pulse sequence parameters as the resting-state session except for number of volumes. It consisted of 10 interleaved blocks of task and rest, with a block length of 30 s. In total, 300 volumes were acquired in a scan time of $10 \mathrm{~min}$ and $6 \mathrm{~s}$. For detailed description of the stimulation paradigm, see Checkerboard stimulation.

\section{Checkerboard stimulation}

During the openSTIM condition of MRS and fMRI acquisitions in the main study cohort, a checkerboard stimulation was presented to the participants. In a task block, a full-screen size black-and-white flickering 

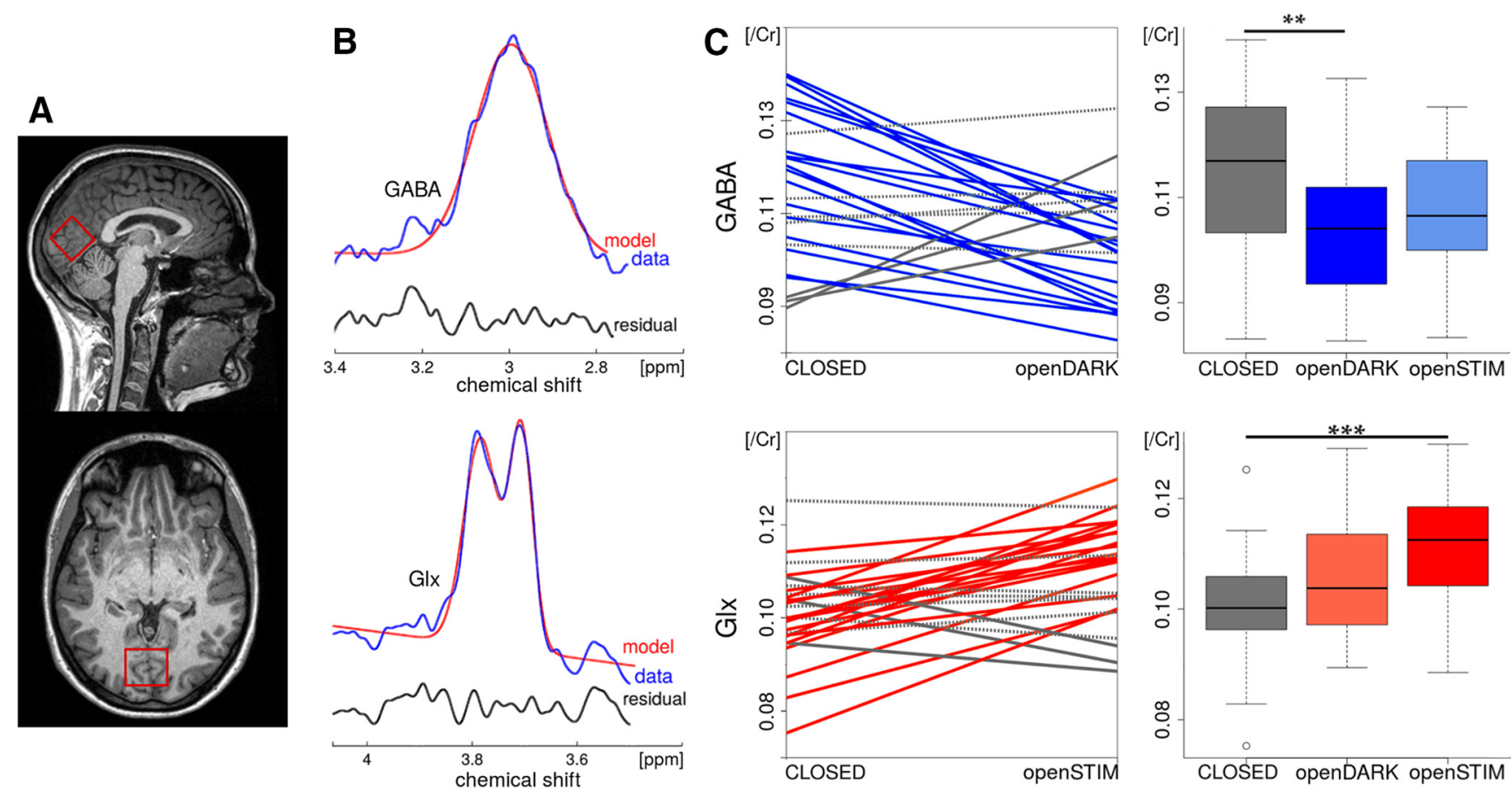

Figure 2. MRS measurements and results. A, MRS voxel placement in the occipital cortex in the sagittal and axial planes. $B$, Representative fits of the GABA+ and Glx peaks in the edited MEGA-PRESS spectrum. C, Creatine-normalized levels of metabolites in the visual cortex in CLOSED, openDARK, and openSTIM. Left, Changes of GABA+/Cr and Glx/Cr between conditions in individual subjects. Colored lines indicate $\geq 5 \%$ differences in metabolite-to-creatine ratios coherent with the statistically significant results on the group level. Gray solid lines indicate $\geq 5 \%$ difference in the opposite direction. Gray dotted lines indicate weaker change. Right, Box plots of relative metabolite levels for each group. Statistically significant difference: ${ }^{* *} p<0.01 ;{ }^{* * *} p<$ 0.001 .

checkerboard was displayed with a flicker frequency of $8 \mathrm{~Hz}$. During a rest block, a black fixation cross on a white background was presented. Before each run, participants were instructed to focus and pay attention to either the flickering checkerboard or the cross. Stimulation was presented on a BOLD Screen 32 MR Safe Display (Cambridge Research Systems) with a resolution of $1920 \times 1080$ and a frame rate of $120 \mathrm{~Hz}$. The screen was placed in the back of the scanner's bore. Participants viewed the presentation on a mirror installed on the coil.

\section{Behavioral experiments}

The behavioral data consisted of two experiments: an orientation discrimination task testing early visual processing and an attentional capture task as a control condition. We used these tasks to relate GABA and Glu signals in the early visual cortex to the processing accuracy of simple visual stimuli while controlling for attentional modulation (de Fockert et al., 2004). Throughout the testing, participants were required to have normal or corrected-to-normal vision. All except 2 volunteers from the main cohort took part in the behavioral experiments.

\section{Orientation discrimination experiment}

Apparatus. The gaze position was recorded using an EyeLink 1000 Desktop Mount (SR Research) at a sampling rate of $1 \mathrm{kHz}$. Participants' head movements were minimized using a chin and forehead rest. The experiment was controlled by an iMac Intel Core i5 computer (Apple). The experimental software was implemented in MATLAB (The MathWorks, RRID:SCR_001622), using the Psychophysics (Brainard, 1997; Pelli, 1997) (RRID:SCR_002881) and EyeLink (Johns et al., 2007) (RRID: SCR_009602) toolboxes. Stimuli were presented at a viewing distance of $60 \mathrm{~cm}$ on a 21 inch gamma-linearized GDM-F500R CRT screen (SONY) with a spatial resolution of $1024 \times 768$ pixels and a vertical refresh rate of $120 \mathrm{~Hz}$.

Procedure. The orientation discrimination design was adapted from Edden et al. (2009) and modified according to Hanning et al. (2017). Subjects initially fixated at a central black fixation $\operatorname{dot}\left(0 \mathrm{~cd} / \mathrm{m}^{2}, 0.15^{\circ}\right.$ radius) on a gray background $\left(30 \mathrm{~cd} / \mathrm{m}^{2}\right)$ (see Fig. $4 A$ ). Once a stable fixation was detected within a $2.0^{\circ}$ radius around the fixation dot, a flickering Gabor/noise stream appeared at the fixation. The stimulus was composed of interleaved sequences of vertically oriented Gabor patches (frequency 2.5 cpd; random phase; $100 \%$ contrast; SD of Gaussian envelope $1.1^{\circ}$; mean luminance $30 \mathrm{~cd} / \mathrm{m}^{2}$ ) and Gaussian pixel noise patches $\left(0.22^{\circ}\right.$-width pixels ranging from white $(1)$ to black $(0)$; the same Gaussian envelope as for the Gabors), alternating every $25 \mathrm{~ms}$. After 400-800 $\mathrm{ms}$, this stream contained a discrimination Gabor patch rotated clockwise or counterclockwise. Following the constant stimuli method, tilt angles (nine linearly spaced angles from $1^{\circ}$ to $17^{\circ}$ ) were varied randomly across trials. After $25 \mathrm{~ms}$, the Gabor patch was masked by another noise patch. The Gabor/noise stream disappeared after $200 \mathrm{~ms}$, and participants indicated via button press in a nonspeeded manner whether the discrimination Gabor was tilted clockwise or counterclockwise. They received a negative feedback sound for incorrect responses. After one practice block, each participant performed seven blocks of 35 trials. We controlled online for broken fixation (not within $2.0^{\circ}$ from the fixation dot) and repeated erroneous trials in random order at the end of each block.

\section{Attentional capture experiment}

Stimuli and design. The attentional capture paradigm, adapted from Theeuwes (1992), was implemented in the Open Sesame software version 3.1.6b1 (Mathôt et al., 2012), modified and run on an Intel computer. The visual display comprised of 8 circular $\left(1.4^{\circ}\right.$ in diameter $)$ or rectangular geometrical stimuli (100 pixels). Yellow $\left(97.6 \mathrm{~cd} / \mathrm{m}^{2}\right)$ and blue $\left(12.3 \mathrm{~cd} / \mathrm{m}^{2}\right)$ shapes were displayed on a black background $\left(1.24 \mathrm{~cd} / \mathrm{m}^{2}\right)$. In each trial, one stimulus represented a unique shape (e.g., seven circular, one rectangular). The target, a white line segment $\left(111 \mathrm{~cd} / \mathrm{m}^{2}, 75\right.$ pixels), was presented inside a unique shape with either a vertical or horizontal orientation (with possible options: $0^{\circ}$ or $90^{\circ}$ ). Other line segments inside nontarget figures were tilted either to the right or the left (possible orientations: $30^{\circ}, 60^{\circ}, 120^{\circ}, 150^{\circ}$ ) and distributed randomly on the display. In distractor-absent trials, all shapes were presented in the same color (e.g., seven circular shapes and one rectangular shape, all blue). In distractor-present trials, one of the blue circles was replaced with a yellow circle. Participants were asked to indicate the orientation of 
the line segment in the unique shape. The experiment consisted of 7 blocks of 20 trials each (140 trials in total). A chin rest was used to stabilize the observer's head at a distance of $55 \mathrm{~cm}$ from the monitor.

Procedure. After receiving written and oral instructions on the experimental procedure, the experiment was started with a block of practice session (20 trials) to familiarize participants with the task. Next, the remaining six experimental blocks were performed. At the beginning of each trial, a black fixation dot at the center of the screen was presented for $500 \mathrm{~ms}$. The stimuli display was presented subsequently and remained displayed for $3000 \mathrm{~ms}$. Subjects were instructed to respond as quickly and as accurately as possible, and to indicate the line orientation in the unique shape as vertical or horizontal. After an erroneous response, a red dot was presented at the location of the fixation dot for $500 \mathrm{~ms}$.

\section{Data analysis}

$M R S$

MEGA-PRESS spectra were analyzed using the Gannet 2.0 software (Edden et al., 2014) (RRID:SCR_016049). Preprocessing steps included the following: frequency and phase correction in the time domain, $3 \mathrm{~Hz}$ exponential line broadening, and fitting of the choline and creatine signals. After subtracting OFF from ON acquisitions, a single GABA+ peak at $3 \mathrm{ppm}$ (GABA peak with a contribution from macromolecule signals) and a double Glx peak at $3.75 \mathrm{ppm}$ were separately fitted using a fiveparameter Gaussian model. Levels of GABA+ and Glx were calculated from the respective model peak areas, and normalized by the area of the creatine peak model.

Next, the quality control of all acquisitions was performed. In the main study cohort $(N=30)$, from 90 spectra acquired in three conditions, four were excluded because either GABA or Glx could not be fit. One additional spectrum was excluded as it was qualified as noisy (by visual inspection), and it exceeded 3 SDs from the group mean in the Glx FWHM parameter. In the replication cohort $(N=25)$, three complete datasets were excluded ( 6 of 50 spectra) because either GABA or Glx could not be fit. Further analyses performed on the two subgroups revealed that no further datasets exceeded 3 SDs from the group mean in any of the following parameters: GABA+, Glx or Cr fit error, GABA+ FWHM, and Glx FWHM. Plots depicting quality control parameters of all acquisitions can be found in Figure 1.

The "edit-off" spectra were analyzed with the LCModel version 6.3-1 (Provencher, 1993) (RRID:SCR_014455). A simulated basis set was provided by the software developer and included following metabolites: alanine, aspartate, creatine (Cr), phosphocreatine (PCr), GABA, glucose, Glu, Gln, glycerophosphocholine (GPC), phosphocholine (PCh), glutathione, myo-inositol (Ins), lactate, $N$-acetylaspartate (NAA), $N$-acetylaspartylGlu (NAAG), scyllo-inositol, and taurine. Ratios of metabolites to total creatine signal $(\mathrm{Cr}+\mathrm{PCr})$ were calculated. Spectra with peak fitting errors (Cramer-Rao lower bounds) exceeding 20\% were removed from further analysis and the concentration of standard neurometabolites was assessed between conditions: Ins, total phosphocholine $(\mathrm{GPC}+\mathrm{PCh})$ and total $\mathrm{N}$-acetylaspartate (NAA+NAAG).

\section{$f M R I$}

fMRI data were processed using the DPARSF tool (RRID:SCR_002372) and SPM8 (RRID:SCR_007037) functions (Wellcome Trust Center for Neuroimaging, University College London; www.fil.ion.ucl.ac.uk/spm) (Chao-Gan and Yu-Feng, 2010; Song et al., 2011), all implemented in MATLAB. Preprocessing included the following steps: slice timing correction, realignment, and reorientation. Participants did not exhibit significant head movements as none of them exceeded $>3 \mathrm{~mm}$ of maximal translation and 3 degrees of maximal rotation of overall estimated head motion during the course of the experiment. After the coregistration of the structural image to the functional mean volume, the structural volume was segmented, and the mean white matter and CSF signals were regressed from the functional signal.

Amplitude of low-frequency fluctuations (ALFF) in resting-state CLOSED and openDARK conditions. The preprocessed AMRI data were smoothed with a $4 \mathrm{~mm}$ Gaussian kernel, linearly detrended, and kept in the subjects' native space without normalization. The signal time series from each voxel in the frequency range of $0.01-0.1 \mathrm{~Hz}$ were transformed into the frequency domain. The square root of each frequency was calculated and averaged (Zang et al., 2007). Standardized $z$ score ALFF maps were derived and used for later analyses. To extract the fMRI scores from the voxel of interest corresponding to the individually placed MRS voxel, a binary mask representing the MRS voxel was created with the Gannet 2.0 software. The matrix derived from the coregistration of the structural image to the mean functional volume was used to coregister the binary MRS voxel to the fMRI data space. The zALFF scores were extracted and averaged across the voxels of interest. The difference in zALFF between the CLOSED and openDARK conditions was assessed using a two-tailed paired-samples $t$ test. Pearson product-moment correlation coefficients were computed between the individual zALFF scores and the MRSderived GABA+ and Glx levels.

$B O L D$ signal change in openSTIM condition. After preprocessing, the structural and functional images were coregistered, normalized to MNI space, and smoothed using a $6 \mathrm{~mm}$ FWHM Gaussian kernel. To analyze statistical differences between blocks of task and rest conditions, 10 interleaved blocks of ON and OFF were modeled using the SPM canonical hemodynamic response function with motion parameters added to the general linear model. A family-wise error (FWE) correction was used $(p<0.001$ height, $p<0.05$ extent threshold) for the mass-univariate voxel-wise testing. The contrast of interest was defined as $\left(\beta_{\text {visual }}-\beta_{\text {rest }}\right)$. The contrast estimates were extracted from the individuals' peak voxels located in the visual cortex and were later used for analysis. At this stage, one participant had to be excluded due to a lack of significant activation clusters (FWE-corrected) in the visual cortex. Pearson product-moment correlation coefficients were computed between the contrast estimates and GABA+ and Glx levels.

\section{Orientation discrimination task}

By fitting cumulative Gaussian functions to each subject's performance, the tilt angle corresponding to $75 \%$ correct performance in orientation discrimination was determined and averaged to $7.1^{\circ}\left(\mathrm{SD}=4.18^{\circ}\right.$, minimum $=2.14$, maximum $=17.03)$. The obtained discrimination thresholds were taken as a proxy of visual sensitivity and correlated with each participants' GABA + and Glx levels using the Pearson product-moment correlation. Five participants were unable to discriminate any of the tilt angles above chance level (50\% correct), most probably because they could not see the oriented Gabor patch that was presented very briefly ( 25 $\mathrm{ms}$ ) without extensive training. Their data were excluded from further analysis.

\section{Attentional capture task}

After excluding erroneous trials from the analysis, the mean reaction times (RT) and the accuracy of all trials were analyzed. Overall task response accuracy averaged to $94.0 \%$. Error rates were equivalent for both: distractor-absent and -present conditions and comprised of 15 erroneous trials. The mean RT of a distractor absent trial was $986 \mathrm{~ms}$, and the mean RT of a distractor present trial was $1127 \mathrm{~ms}$. A paired-samples one-tailed $t$ test was conducted to compare differences in response RTs between the distractor-present and distractor-absent trials and revealed a significant difference between the two conditions $\left(t_{(27)}=8.38, p=\right.$ 0.001 ). Pearson product-moment correlation coefficients were computed to assess the relationship between the mean RT values of distractor absent and distractor present trials in the attentional capture task and the neurotransmitter levels.

\section{Experimental design and statistical analysis}

To assess the differences in metabolite concentrations between the three conditions, a hierarchical testing procedure was conducted. In the first step, ANOVA for repeated measurements was performed to test the global null hypothesis of equal means under all conditions on a significance level of $5 \%$. If the global null hypothesis was rejected, pairwise comparisons of conditions were performed on an unadjusted level of significance. The difference in GABA+/Cr levels between the CLOSED and openDARK conditions found in main study cohort was reproduced in the replication cohort with the pairwise comparisons test. 
In addition to analyzing differences in metabolite levels between conditions, we also assessed unspecific variations in MRS signals by controlling the following parameters: FWHM of GABA+ and Glx peaks (with ANOVAs), correlations between GABA+ and Glx levels (with the Pearson product-moment correlation coefficients), and the normality of the group data (with Shapiro-Wilk tests).

Finally, individual MRS data were correlated with fMRI scores (ALFF and BOLD signal change) and behavioral scores in linear regression analyses.

\section{Results}

\section{GABA levels decrease whereas Glx levels increase with} increasing visual input

We first investigated changes in neurotransmitter levels between the states of CLOSED, openDARK, and openSTIM $(N=25)$ (Table 2). We found a significant GABA+/Cr decrease from CLOSED $(0.12 \pm 0.02)$ to openDARK $(0.10 \pm 0.01)$ (ANOVA: $F_{(2,72)}=4.77, p=0.013$; post hoc paired-samples two-tailed $t$ test: $\left.t_{(24)}=-3.14, p=0.004\right)$ with no further change in openSTIM ( post hoc paired-samples two-tailed $t$ test: $t_{(24)}=-1.78, p=0.09$ ) (Fig. 1C). Glx/Cr increased from CLOSED $(0.10 \pm 0.01)$ to openSTIM $(0.11 \pm 0.01)\left(\right.$ ANOVA: $F_{(2,72)}=6.76, p=0.003$; post hoc paired-samples two-tailed $t$ test: $\left.t_{(24)}=4.03, p=0.0005\right)$ and shown a trend toward an increase in openDARK (post hoc pairedsamples two-tailed $t$ test: $t_{(24)}=2.16, p=0.04$ ) (Fig. 1C). After including the data from the replication cohort $(N=22)$, we reproduced the significant difference in GABA+/Cr between CLOSED $(0.12 \pm 0.02)$ and openDARK $(0.11 \pm 0.01)$ (pairedsamples one-tailed $t$ test on the replication cohort data: $t_{(21)}=$ $-1.58, p=0.06$; paired-samples one-tailed $t$ test on the data from both cohorts: $\left.t_{(47)}=-3.37, p=0.0007\right)$.

In summary, GABA + levels dropped from the CLOSED to openDARK state with no further change during the visual input processing in openSTIM, whereas Glx levels did not differ between the two baseline states of eyes CLOSED and openDARK (in darkness) but increased with visual input (openSTIM).

Control analyses revealed no effects of the state of visual input on FWHM $(\mathrm{GABA}+)\left(F_{(2,72)}=1.13, p=0.33\right)$ or on FWHM $(\mathrm{Glx})\left(F_{(2,72)}=0.61, p=0.54\right)$ (Table 3$)$. Also, GABA $+/ \mathrm{Cr}$ and $\mathrm{Glx} / \mathrm{Cr}$ group results did not violate the normality of the distribution (group W scores and $p$ values can be found in Table 4). Finally, GABA+/Cr and Glx/Cr did not correlate within conditions ( $r$ and $p$ values can be found in Table 5).

In summary, the control analyses revealed no changes in GABA + and Glx linewidth stability nor in the normality of the distribution between conditions. Additionally, no significant correlation was found between the neurotransmitter levels across conditions, suggesting that the measurements of both signals are independent.

Last, we tested for the specificity of the visual state changes on GABA + and Glx levels by assessing other metabolite levels in the "edit-off" spectrum. The levels of Ins, GPC+PCh, and $\mathrm{NAA}+\mathrm{NAAG}$ did not differ between conditions $(p<0.05)$, suggesting selective changes across states only for inhibitory and excitatory neurotransmitters. The averaged metabolite levels and detailed statistics can be found in Table 6 .

\section{GABA levels correlate negatively with neural signaling only in openDARK}

In addition to edited MRS, we acquired fMRI data during identical states of visual input and calculated local neuronal activity with BOLD-signal amplitude during CLOSED/openDARK (zALFF) and openSTIM (\% signal changes). We tested for differences in the zALFF signal averaged across the MRS voxel with a
Table 2. Group-averaged GABA+/Cr and Glx/Cr ratios in CLOSED, openDARK, and openSTIM derived from edited MEGA-PRESS acquisition and analyzed with Gannet ${ }^{a}$

\begin{tabular}{llll}
\hline Group & CLOSED (SD) & openDARK (SD) & openSTIM (SD) \\
\hline GABA+/Cr group averages & & & \\
$\quad$ Main cohort $(N=25)$ & $0.115(0.016)$ & $0.103(0.012)$ & $0.107(0.012)$ \\
$\quad$ Both cohorts $(n=47)$ & $0.115(0.015)$ & $0.106(0.012)$ & - \\
Glx/Cr group averages & & & \\
$\quad$ Main cohort $(n=25)$ & $0.100(0.009)$ & $0.105(0.011)$ & $0.110(0.011)$ \\
$\quad$ Both cohorts $(n=47)$ & $0.094(0.013)$ & $0.096(0.015)$ & - \\
\hline${ }^{a}$ Data are mean (SD). & & &
\end{tabular}

Table 3. Group-averaged FWHM scores of GABA + and Glx peaks in CLOSED, openDARK and openSTIM ${ }^{a}$

\begin{tabular}{llll}
\hline Group & CLOSED (SD) & openDARK (SD) & openSTIM (SD) \\
\hline GABA + FWHM & & & \\
$\quad$ Main cohort $(n=25)$ & $18.43(2.20)$ & $18.04(2.25)$ & $19.01(2.18)$ \\
$\quad$ Both cohorts $(n=47)$ & $18.63(2.06)$ & $18.04(1.90)$ & - \\
Glx FWHM & & & \\
$\quad$ Main cohort $(n=25)$ & $15.16(2.54)$ & $14.66(1.16)$ & $14.87(1.36)$ \\
$\quad$ Both cohorts $(n=47)$ & $14.85(2.18)$ & $14.54(1.22)$ & - \\
\hline
\end{tabular}

${ }^{a}$ Data are mean (SD).

Table 4. Shapiro test results of GABA $+/ \mathrm{Cr}$ and $\mathrm{GIx} / \mathrm{Cr}$ in CLOSED, openDARK, and openSTIM ${ }^{a}$

\begin{tabular}{llll}
\hline Group & CLOSED & openDARK & openSTIM \\
\hline $\begin{array}{l}\text { Shapiro test results for GABA }+/ C r \\
\text { group averages }\end{array}$ & & \\
$\begin{array}{l}\text { Main cohort }(n=25) \\
\text { Both cohorts }(n=47)\end{array}$ & $0.9561, p=0.34$ & $0.9611, p=0.44$ & $0.9689, p=0.62$ \\
Shapiro test results for Glx $/ \mathrm{Cr}$ & & & \\
$\quad$ group averages & & & \\
Main cohort $(n=25)$ & $0.9701, p=0.27$ & $0.9763, p=0.45$ & - \\
Both cohorts $(n=47)$ & $0.9709, p=0.29$ & $0.9877, p=0.89$ & -
\end{tabular}

${ }^{a} \mathrm{~W}$ scores and $p$ values are depicted for each separate group of interest.

Table 5. Correlation coefficients between GABA $+/ \mathrm{Cr}$ and $\mathrm{Glx} / \mathrm{Cr}$ in CLOSED, openDARK and openSTIM ${ }^{a}$

\begin{tabular}{llll}
\hline Group & CLOSED & openDARK & openSTIM \\
\hline Main cohort $(n=25)$ & $-0.392(p=0.054)$ & $-0.133(p=0.53)$ & $-0.106(p=0.61)$ \\
Both cohorts $(n=47)$ & $-0.110(p=0.46)$ & $-0.152(p=0.31)$ & -
\end{tabular}

${ }^{a}$ Values are Pearson correlation coefficients and ( $p$ values).

Table 6. Group-averaged metabolites levels in the "edit-off" spectrum in the CLOSED, openDARK, and openSTIM conditions ${ }^{a}$

\begin{tabular}{lllll}
\hline Metabolite $(/ \mathrm{Cr}+\mathrm{PCr})$ & CLOSED & openDARK & openSTIM & ANOVA $F_{(2,81)}^{b}$ \\
\hline Ins & $0.76(0.076)$ & $0.76(0.078)$ & $0.73(0.069)$ & $F=0.09, p=0.92$ \\
GPC + PCh & $0.15(0.013)$ & $0.15(0.014)$ & $0.14(0.011)$ & $F=0.03, p=0.98$ \\
NAA + NAAG & $1.68(0.101)$ & $1.68(0.099)$ & $1.71(0.086)$ & $F=0.04, p=0.96$ \\
\hline
\end{tabular}

${ }^{a}$ Data are mean (SD).

${ }^{b}$ A one-way between subjects ANOVA scores comparing metabolite levels in three conditions.

paired-sample two-tailed $t$ test and found no changes between CLOSED $(0.69 \pm 0.56)$ and openDARK $(0.77 \pm 0.39)\left(t_{(53)}=\right.$ $1.01, p=0.31)$. Voxel distribution of zALFF scores in both states can be found in Figure $3 A$. Further, a group analysis of BOLDsignal changes in openSTIM revealed a significant activation in the primary visual cortex when observing the flickering checkerboard presentation compared with rest $(p<0.05$, FWEcorrected) (Fig. 3B; Table 7).

We further found a significant negative correlation between GABA+/Cr and zALFF scores in openDARK $(r=-0.35, n=48$, 


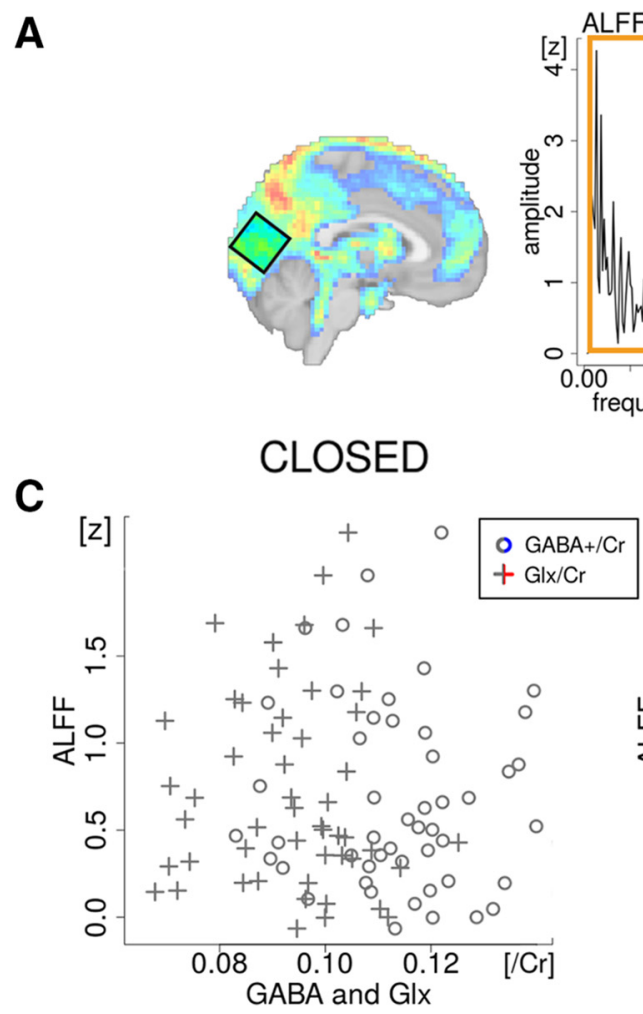

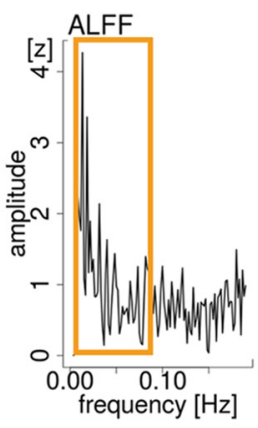

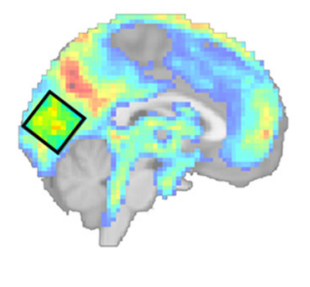

openDARK

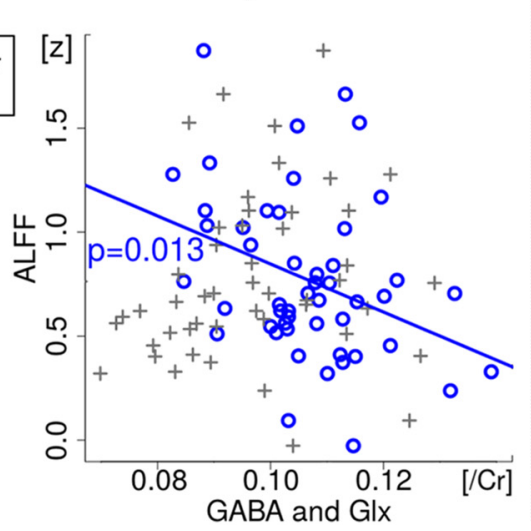

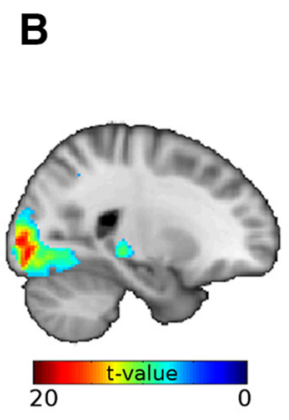
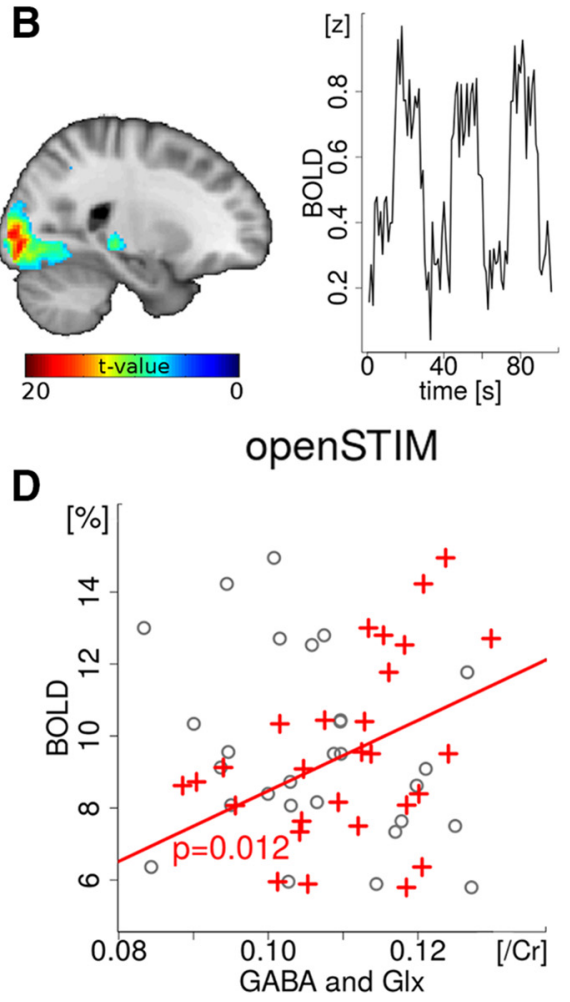

Figure 3. Local neural activity and Cr-normalized metabolite levels. $\boldsymbol{A}$, Regional distribution of ZALFF scores in CLOSED and openDARK states. ALFF is calculated as the sum of amplitudes in the power spectrum of BOLD signal between the frequency of 0.01 and $0.1 \mathrm{~Hz}$. For further analysis, ZALFF signal was averaged across the MRS voxel in the occipital cortex. $\boldsymbol{B}$, Task-related B0LD signal changes in openSTIM. Areas with significant fMRI activation when observing the flickering checkerboard presentation compared with the rest condition ( $p<0.05$, FWE-corrected). The main cluster is located in the primary visual cortex. $C$, Relationship between zALFF scores, GABA $+/ \mathrm{Cr}$, and Glx $/ \mathrm{Cr}$ in CLOSED and openDARK. GABA $+/ \mathrm{Cr}$ correlates negatively with zALFF $(p=0.013)$ in openDARK. D, Relationship between BOLD \%, GABA $+/ \mathrm{Cr}$, and Glx/Cr in openSTIM. Glx/Cr correlates positively $(p=0.012)$ with BOLD signal change.

Table 7. fMRI group results corrected with FWE at $p<0.05$ level $^{a}$

\begin{tabular}{llll}
\hline Anatomical region & MNI coordinates $(x, y, z)$ & $t$ & Cluster size \\
\hline Primary visual cortex & $-22,-98,6$ & 15.41 & 9845 \\
& $-22,-90,8$ & 15.01 & - \\
Primary motor cortex & $-14,-96,-2$ & 14.69 & - \\
Primary sensory cortex & $-44,-10,56$ & 10.50 & 725 \\
& $-58,-10,44$ & 9.03 & - \\
& $40,-26,46$ & 9.39 & 1858 \\
& $58,2,40$ & 9.33 & - \\
\hline
\end{tabular}

${ }^{a}$ Only clusters $>100$ voxels were included. Anatomical regions, MNI coordinates of local maxima, $t$ values, and cluster sizes (in voxels) are depicted.

$p=0.013)$, but neither in CLOSED $(r=0.12, n=51, p=0.41)$ nor in openSTIM $(r=-0.28, n=28, p=0.14)$ (Fig. $2 C, D)$.

In summary, GABA + levels only correlated with local neural signaling in openDARK but not in CLOSED or openSTIM.

\section{Glx levels correlate positively with neural activity in openSTIM}

Next, we investigated whether Glx levels correlated with the BOLD-signal amplitude in different states. The Glx/Cr concentration did not relate to zALFF scores in CLOSED $(r=-0.01$, $n=51, p=0.93)$ or in openDARK $(r=0.16, n=49, p=0.25)$. However, we found a significant positive correlation between $\mathrm{Glx} / \mathrm{Cr}$ and BOLD contrast estimates in openSTIM $(r=0.41, n=$ $28, p=0.012$ ) (Fig. 3C,D).

In summary, Glx levels and fMRI task responses correlate during visual stimulation but not in states without visual input.

\section{GABA levels correlate positively with visual sensitivity}

In a final step, we tested whether neurotransmitter levels in openSTIM related to the efficiency of basic visual processes. The analyses revealed a significant negative correlation between orientation discrimination thresholds and GABA+/Cr in openSTIM $(r=$ $-0.54, n=23, p=0.008)$. That is, participants with a higher GABA concentration during visual stimulation were able to discriminate smaller tilt angles, which we take as an indicator of higher visual sensitivity. No such correlation was observed for GlX/Cr $(r=0.08, n=23, p=0.72)$ (Fig. 4B). Neurotransmitter levels also did not correlate with the performance in the attentional capture task measured as mean RTs of distractor absent and distractor present trials $\left(\mathrm{r}_{\mathrm{GABA}}=0.24, n=27, p=0.28\right.$; $\left.\mathrm{r}_{\mathrm{Glx}}=-0.17, n=27, p=0.44\right)$.

In summary, GABA+, but not Glx, levels during visual stimulation correlated with visual sensitivity in an orientation discrimination task. Neurotransmitter levels were not associated with cognitive modulation of visual processes.

\section{Discussion}

In this study, we investigated whether $\mathrm{GABA}+/ \mathrm{Cr}$ and $\mathrm{Glx} / \mathrm{Cr}$ ratios in the occipital cortex fluctuate with increasing visual input (openDARK and openSTIM) compared with an eyes-closed (CLOSED) condition. As expected, we found contrasting changes and different dynamics of both neurotransmitters. When opening the eyes in darkness, inhibitory GABA levels decreased and correlated negatively with the BOLD-signal amplitude, with no further changes in openSTIM. Glu levels did not differ between CLOSED and openDARK but increased with checkerboard stimulation, where they positively correlated with BOLD signal change. On 


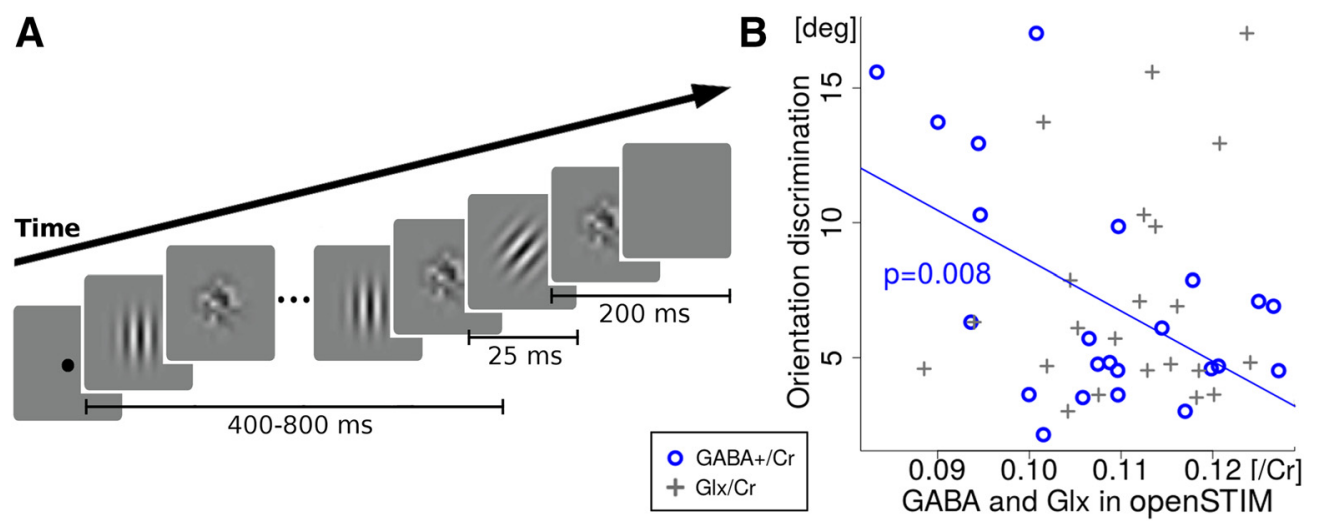

Figure 4. Orientation discrimination task. $A$, Sequence of events in the course of each trial. $B$, Negative correlation between GABA+/Crin openSTIM and orientation discrimination thresholds in Gabor tilt angles. A smaller discrimination threshold corresponds to better performance (i.e., higher visual sensitivity).

the behavioral level, we found that inhibitory but not excitatory neurotransmitter levels in the occipital cortex correlated with visual discrimination performance.

\section{GABA and Glx levels change with increasing visual input}

Previous studies consistently identified upregulation of excitatory neurotransmitter levels during visual processing, whereas reports about GABA changes were less consistent. Mekle et al. (2017) found a 5\% reduction of GABA concentration in the activated hemisphere during unilateral visual stimulation compared with eyes open. Others could not detect such alterations (Mangia et al., 2007; Schaller et al., 2014; Bednarík et al., 2015). Importantly, all these experiments compared levels of high visual input (i.e., presentation of a checkerboard) with low visual input (usually eyes open in a bright scanner environment) but did not include an eyes-closed condition. In the current study, we explicitly contrasted high (openSTIM) and low (openDARK) visual input conditions with eyes closed. This revealed a downregulation of GABA even before receiving visual stimulation. To the best of our knowledge, this is the first report to describe fluctuations in GABA levels induced solely by opening the eyes in the dark. Our results might explain the heterogeneous findings on GABA from prior studies, as these could have missed prominent changes of neurotransmitter levels when only comparing high versus low stimulation periods.

Whereas GABA levels decreased after opening the eyes in the dark, Glx increased in the openSTIM state. This result is in line with previous reports about the fluctuations in excitatory neurotransmitter levels during visual processing. Presentation of a flickering checkerboard in blocks of 1 to $10 \mathrm{~min}$ increased Glu concentration by 2\% to 4\% (Mangia et al., 2007; Lin et al., 2012; Schaller et al., 2014; Bednarík et al., 2015; Ip et al., 2017). In our study, Glx rises by $10 \%$ from CLOSED to openSTIM conditions, exceeding Glu level changes reported previously during visual stimulation (up to 4\%). Several aspects might explain this higher level of changes. First, we compared Glx levels between the state of no external input (CLOSED) and the condition of heavy visual load (openSTIM), whereas others have mainly investigated the differences between openDARK and openSTIM conditions. Second, we acquired the data with edited MEGA-PRESS sequence, whereas others have often used PRESS or STEAM sequences with shorter TEs. Finally, we independently modeled GABA and Glx peaks using Gannet software, whereas others have frequently applied the basis set of LCModel.
In former studies, both prolonged visual stimulation and observation of more discrete visual stimuli were found to elevate levels of Glu. Looking at abstract stimuli causes a stronger rise in the Glu concentration in the lateral occipital cortex than viewing objects (Lally et al., 2014). Observing novel stimuli, compared with those already seen, leads to a further increase of Glu levels in the lateral occipital cortex by 11\%-13\% (Apsvalka et al., 2015). Together with our data, these results suggest that occipital Glu levels increase with the amount of ongoing bottom-up visual processing. In summary, our findings on diverging GABA and Glu signal changes point to the importance of defining proper baseline conditions for both neurotransmitters.

\section{Neurotransmitter levels correlate with the BOLD signal}

We found that, in corresponding conditions, the levels of excitatory and inhibitory neurotransmitters related differently to local neuronal activity in the visual system, as measured with fMRI: GABA levels correlated negatively with the amplitude of BOLD signal fluctuations in the openDARK state, whereas Glx levels correlated positively with the BOLD signal change in the openSTIM state. Prior studies thus far have related baseline GABA (i.e., during eyes open in resting state) with BOLD signal changes during visual stimulation but not in identical conditions. While several studies found a negative correlation between resting GABA and BOLD signal change during stimulation (Muthukumaraswamy et al., 2009; Donahue et al., 2010; Bednarík et al., 2015), others failed to replicate such an association (Harris et al., 2015). Instead of relating MRS and BOLD signals across conditions, we therefore acquired both in identical brain states sequentially and in balanced order.

One way to study spontaneous brain activity at rest is to evaluate ALFF scores, a spectral property of the BOLD signal computed in the low-frequency range (Zang et al., 2007; Zuo et al., 2010). Interactions between neurotransmitter levels and ALFF have thus far not been investigated. Our findings demonstrate that higher levels of GABA in openDARK correlate with a reduced BOLD signal amplitude in the occipital cortex across subjects. This suggests that the local neural activity in the visual system as captured by fMRI is indeed reduced by increased GABA levels. In summary, our results highlight the importance of studying MRS and BOLD relationships during identical functional states rather than across different conditions.

Acquired in the state of receiving strong visual input, Glx levels in the occipital cortex positively correlated with BOLD sig- 
nal changes. Similar relationships were reported in previous studies. Recently, Ip et al. (2017) simultaneously acquired MRS and BOLD signals while stimulating the visual system with a flickering checkerboard. They found a significant positive correlation between the time courses of the two measures, suggesting that visual input triggers both a positive BOLD response as well as higher levels of Glu. However, they did not find such a relationship between resting Glu levels (measured in CLOSED) and BOLD signal change during stimulation. Likewise, in an experiment by Bednarík et al. (2015), consecutively acquired MRS and BOLD signals correlated positively in a visual stimulation condition. Together, these results suggest that higher excitatory neurotransmitter levels predict stronger changes in neural activity in a visual task. It also confirms our observation that both GABA and Glx levels relate to BOLD signal change rather during identical states than across conditions.

\section{GABA levels correlate with behavioral outcomes}

Finally, we found that the levels of GABA, but not Glx, correlated with participants' individual psychophysiological performance. High levels of GABA during visual processing were associated with better behavioral performance in a demanding visual discrimination task. Local GABA, however, did not relate to behavioral performance in a more complex control task that uses attentional modulation. This suggests that GABA in the visual system shapes the precision of early visual processing capacity. In the task, the relevant orientation signal (the clockwise or counterclockwise tilted Gabor) was embedded in a stream of alternating visual noise patches and presented for only $25 \mathrm{~ms}$. Higher local GABA might therefore help to tune the reactiveness to the target stimulus or, in general, decrease the reactivity of the visual system to irrelevant input. Other studies found that higher resting GABA concentration in the occipital cortex was associated with better orientation discrimination performance (Edden et al., 2009) and facilitated the suppression of orientation-specific features (Yoon et al., 2010). The essential role of GABAergic signaling in establishing selectivity of the visual system to certain features was also demonstrated in animal studies: blocking GABA receptors in a cat's visual system with the antagonist gabazine increased its responsiveness to visual stimulation by $300 \%$ but decreased the selectivity for stimulus orientation and direction (Katzner et al., 2011). In humans, similar evidence for an effect of GABA on behavioral performance was observed in the motor system: Puts et al. (2011) found that higher GABA levels in the sensorimotor cortex associate with higher scores in a tactile frequency discrimination task. Together, these results indicate that local GABAergic inhibition regulates the fine-tuning of a functional system to improve the behavioral outcome.

\section{Limitations of edited MRS measurements}

The signal differences we observed in GABA and Glx levels between conditions might have been caused by unspecific changes in spectral parameters. To exclude those effects, we evaluated the parameters of all acquisitions. We found no alterations in the width (FWHM) of either spectra between conditions, and GABA and Glx signals did not correlate with each other. Furthermore, the change in the metabolite concentration was specific for the two molecules of interest, as other metabolites did not fluctuate between conditions. Consequently, our control analyses suggest that MRS independently captures both neurotransmitters and that changes were related to physiological differences across brain states.
The GABA + levels quantified in our edited MRS experiment also contain additional macromolecule components (Mullins et al., 2014). In this study, however, it is not a major confounder, as we compare GABA+ levels across different functional states of the visual system. Macromolecule signals should be stable between conditions; therefore, we can attribute GABA+ fluctuations to GABA level changes. The physiological interpretation of found GABA decrease exceeds the scope of this study. Edited MRS is not capable of separating extrasynaptic and intrasynaptic GABA pools and rather reflects a bulk GABA cellular signal than synaptic GABA activity (Rae, 2014; Stagg et al., 2014). Therefore, whether the fluctuations we observed depict alterations in inhibitory transmission remains unclear and needs further research. Last, the Glx signals we report represent mixed concentrations of Glu and Gln. As showed recently by Sanaei et al. (2018), Glu and Gln can be reliably quantified from GABA-edited MEGA-PRESS acquisitions. Because the Glx signals acquired in the occipital cortex represent a ratio of Glu/Gln between 2.53 and 3.42 (González de la Aleja et al., 2013; Sanaei et al., 2018), we can conclude that the Glx levels primarily reflect glutamatergic processes.

Note Added in Proof: References to a 'Movie 1' were incorrectly included in the Early Release version published October 3, 2018. References to the movie file have now been removed.

\section{References}

Apsvalka D, Gadie A, Clemence M, Mullins PG (2015) Event-related dynamics of glutamate and BOLD effects measured using functional magnetic resonance spectroscopy (fMRS) at $3 \mathrm{~T}$ in a repetition suppression paradigm. Neuroimage 118:292-300. CrossRef Medline

Bednarík P, Tkác I, Giove F, DiNuzzo M, Deelchand DK, Emir UE, Eberly LE, Mangia S (2015) Neurochemical and BOLD responses during neuronal activation measured in the human visual cortex at 7 tesla. J Cereb Blood Flow Metab 35:601-610. CrossRef Medline

Brainard DH (1997) The psychophysics toolbox. Spat Vis 10:433-436. CrossRef Medline

Chao-Gan Y, Yu-Feng Z (2010) DPARSF: a MATLAB toolbox for "pipeline" data analysis of resting-state fMRI. Front Syst Neurosci 4:13. CrossRef Medline

Cousijn H, Haegens S, Wallis G, Near J, Stokes MG, Harrison PJ, Nobre AC (2014) Resting GABA and glutamate concentrations do not predict visual gamma frequency or amplitude. Proc Natl Acad Sci U S A 111:93019306. CrossRef Medline

de Fockert J, Rees G, Frith C, Lavie N (2004) Neural correlates of attentional capture in visual search. J Cogn Neurosci 16:751-759. CrossRef Medline

Donahue MJ, Near J, Blicher JU, Jezzard P (2010) Baseline GABA concentration and fMRI response. Neuroimage 53:392-398. CrossRef Medline

Edden RA, Muthukumaraswamy SD, Freeman TC, Singh KD (2009) Orientation discrimination performance is predicted by GABA concentration and gamma oscillation frequency in human primary visual cortex. J Neurosci 29:15721-15726. CrossRef Medline

Edden RA, Puts NA, Harris AD, Barker PB, Evans CJ (2014) Gannet: a batch-processing tool for the quantitative analysis of gammaaminobutyric acid-edited MR spectroscopy spectra. J Magn Reson Imaging 40:1445-1452. CrossRef Medline

González de la Aleja J, Ramos A, Mato-Abad V, Martínez-Salio A, Hernández-Tamames JA, Molina JA, Hernández-Gallego J, AlvarezLinera J (2013) Higher glutamate to glutamine ratios in occipital regions in women with migraine during the interictal state. Headache 53:365375. CrossRef Medline

Hanning NM, Deubel H, Szinte M (2017) Sensitivity measures of visuospatial attention. J Vis 17:673. CrossRef

Harris AD, Glaubitz B, Near J, Evans JC, Puts NA, Schmidt-Wilcke T, Tegenthoff M, Barker PB, Edden RA (2014) Impact of frequency drift on gamma-aminobutyric acid-edited MR spectroscopy. Magn Res Med 72: 941-948. CrossRef Medline

Harris AD, Puts NA, Anderson BA, Yantis S, Pekar JJ, Barker PB, Edden RA (2015) Multi-regional investigation of the relationship between func- 
tional MRI blood oxygenation level dependent (BOLD) activation and GABA concentration. PLoS One 10:e0117531. CrossRef Medline

Ip IB, Berrington A, Hess AT, Parker AJ, Emir UE, Bridge H (2017) Combined fMRI-MRS acquires simultaneous glutamate and BOLD-fMRI signals in the human brain. Neuroimage 155:113-119. CrossRef Medline

Johns MW, Tucker A, Chapman R, Crowley K, Michael N (2007) Monitoring eye and eyelid movements by infrared reflectance oculography to measure drowsiness in drivers. Somnologie 11:234-242. CrossRef

Katzner S, Busse L, Carandini M (2011) $\mathrm{GABA}_{\mathrm{A}}$ inhibition controls response gain in visual cortex. J Neurosci 31:5931-5941. CrossRef Medline

Lally N, Mullins PG, Roberts MV, Price D, Gruber T, Haenschel C (2014) Glutamatergic correlates of gamma-band oscillatory activity during cognition: a concurrent ER-MRS and EEG study. Neuroimage 85:823-833. CrossRef Medline

Lin Y, Stephenson MC, Xin L, Napolitano A, Morris PG (2012) Investigating the metabolic changes due to visual stimulation using functional proton magnetic resonance spectroscopy at $7 \mathrm{~T}$. J Cereb Blood Flow Metab 32:1484-1495. CrossRef Medline

Mangia S, Tkác I, Gruetter R, Van de Moortele PF, Maraviglia B, Uğurbil K (2007) Sustained neuronal activation raises oxidative metabolism to a new steady-state level: evidence from $1 \mathrm{H}$ NMR spectroscopy in the human visual cortex. J Cereb Blood Flow Metab 27:1055-1063. CrossRef Medline

Mathôt S, Schreij D, Theeuwes J (2012) OpenSesame: an open-source, graphical experiment builder for the social sciences. Behav Res Methods 44:314-324. CrossRef Medline

Mekle R, Kuehn S, Pfeiffer H, Aydin S, Schubert F, Ittermann B (2017) Detection of metabolite changes in response to a varying visual stimulation paradigm using short-TE 1H MRS at 7T. NMR Biomed 30:e3672. CrossRef Medline

Mullins PG, McGonigle DJ, O'Gorman RL, Puts NA, Vidyasagar R, Evans CJ, Edden RA (2014) Current practice in the use of MEGA-PRESS spectroscopy for the detection of GABA. Neuroimage 86:43-52. CrossRef Medline

Muthukumaraswamy SD, Edden RA, Jones DK, Swettenham JB, Singh KD (2009) Resting GABA concentration predicts peak gamma frequency and fMRI amplitude in response to visual stimulation in humans. Proc Natl Acad Sci U S A 106:8356-8361. CrossRef Medline

Pelli DG (1997) The VideoToolbox software for visual psychophysics: transforming numbers into movies. Spat Vis 10:437-442. CrossRef Medline
Provencher SW (1993) Estimation of metabolite concentrations from localized in vivo proton NMR spectra. Magn Res Med 30:672-679. CrossRef Medline

Puts NA, Edden RA, Evans CJ, McGlone F, McGonigle DJ (2011) Regionally specific human GABA concentration correlates with tactile discrimination thresholds. J Neurosci 31:16556-16560. CrossRef Medline

Rae CD (2014) A guide to the metabolic pathways and function of metabolites observed in human brain $1 \mathrm{H}$ magnetic resonance spectra. Neurochem Res 39:1-36. CrossRef Medline

Sanaei NF, Anton A, Michou E, Jung JY, Parkes LM, Williams SR (2018) Quantification of GABA, glutamate and glutamine in a single measurement at $3 \mathrm{~T}$ using GABA-edited MEGA-PRESS. NMR Biomed 31:1. CrossRef Medline

Schaller B, Xin L, O’Brien K, Magill AW, Gruetter R (2014) Are glutamate and lactate increases ubiquitous to physiological activation? A $1 \mathrm{H}$ functional MR spectroscopy study during motor activation in human brain at 7 tesla. Neuroimage 93:138-145. CrossRef Medline

Song XW, Dong ZY, Long XY, Li SF, Zuo XN, Zhu CZ, He Y, Yan CG, Zang YF (2011) REST: a Toolkit for resting-state functional magnetic resonance imaging data processing. PLoS One 6:e25031. CrossRef Medline

Stagg CJ, Bachtiar V, Amadi U, Gudberg CA, Ilie AS, Sampaio-Baptista C, O'Shea J, Woolrich M, Smith SM, Filippini N, Near J, Johansen-Berg H (2014) Local GABA concentration is related to network-level resting functional connectivity. ELife 2014:e01465. CrossRef Medline

Taylor M (2014) MRS of psychiatric disorders. In: Magnetic resonance spectroscopy (Stagg CJ, Rothman D, eds), pp 222-228. San Diego: Academic.

Theeuwes J (1992) Perceptual selectivity for color and form. Percept Psychophys 51:599-606. CrossRef Medline

Yoon JH, Maddock RJ, Rokem A, Silver MA, Minzenberg MJ, Ragland MJ, Carter CS (2010) Gamma-aminobutyric acid concentration is reduced in visual cortex in schizophrenia and correlates with orientation-specific surround suppression. J Neurosci 30:3777-3781. CrossRef Medline

Zang YF, He Y, Zhu CZ, Cao QJ, Sui MQ, Liang M, Tian LX, Jiang TZ, Wang YF (2007) Altered baseline brain activity in children with ADHD revealed by resting-state functional MRI. Brain Dev 29:83-91. CrossRef Medline

Zuo XN, Di Martino A, Kelly C, Shehzad ZE, Gee DG, Klein DF, Castellanos FX, Biswal BB, Milham MP (2010) The oscillating brain: complex and reliable. Neuroimage 49:1432-1445. CrossRef Medline 